\title{
TU/e EmonOWEN

\section{On the potential of lifted domain feedforward controllers with a periodic sampling sequence}

\section{Citation for published version (APA):}

van Zundert, J., Oomen, T. A. E., Goswami, D., \& Heemels, W. P. M. H. (2016). On the potential of lifted domain feedforward controllers with a periodic sampling sequence. In 2016 American Control Conference (ACC 2016), July 6-8, 2016, Boston, MA, USA (pp. 4227-4332). Institute of Electrical and Electronics Engineers. https://doi.org/10.1109/ACC.2016.7525586

DOI:

10.1109/ACC. 2016.7525586

Document status and date:

Published: 01/07/2016

\section{Document Version:}

Accepted manuscript including changes made at the peer-review stage

\section{Please check the document version of this publication:}

- A submitted manuscript is the version of the article upon submission and before peer-review. There can be important differences between the submitted version and the official published version of record. People interested in the research are advised to contact the author for the final version of the publication, or visit the $\mathrm{DOI}$ to the publisher's website.

- The final author version and the galley proof are versions of the publication after peer review.

- The final published version features the final layout of the paper including the volume, issue and page numbers.

Link to publication

\section{General rights}

Copyright and moral rights for the publications made accessible in the public portal are retained by the authors and/or other copyright owners and it is a condition of accessing publications that users recognise and abide by the legal requirements associated with these rights.

- Users may download and print one copy of any publication from the public portal for the purpose of private study or research.

- You may not further distribute the material or use it for any profit-making activity or commercial gain

- You may freely distribute the URL identifying the publication in the public portal.

If the publication is distributed under the terms of Article 25fa of the Dutch Copyright Act, indicated by the "Taverne" license above, please follow below link for the End User Agreement:

www.tue.nl/taverne

Take down policy

If you believe that this document breaches copyright please contact us at:

openaccess@tue.nl

providing details and we will investigate your claim. 


\title{
On the Potential of Lifted Domain Feedforward Controllers with a Periodic Sampling Sequence
}

\author{
Jurgen van Zundert, Tom Oomen, Dip Goswami, and W.P.M.H. Heemels - ACC2016_v99(31/05/2018)
}

\begin{abstract}
Motion control applications traditionally operate with a single-rate, equidistant sampling scheme. For cost reasons, a current trend in industry is consolidating multiple applications on a single embedded platform. Generally, to deal with inter-application interference, a predictable scheduling policy allocates resource to the applications in these platforms. Realizing an equidistant sampling scheme on such shared platform is inflexible and often turns out to be expensive in terms of resource or conservative in terms of performance. The aim of this paper is to investigate the possibilities to relax the equidistant sampling convention. To this end, recent results show that platform timing properties can be represented by a known, precise, and periodically varying set of sampling periods. In view of such predictable platforms, a framework is presented for analysis and synthesis of lifted domain feedforward controllers for periodically time-varying closed-loop systems. Through simulations the potential of such periodically time-varying sampling over conservative equidistant sampling schemes is demonstrated.
\end{abstract}

\section{INTRODUCTION}

Traditional motion controllers are often designed and implemented using a single sampling frequency under equidistant sampling, either in a continuous time with a posterior discretization, a discrete time, or a sampled-data setting [5]. Hence, it is tacitly assumed that resources (i.e., computation, communication, and memory) are sufficiently available.

In certain applications, increasing performance requirements and enhanced functionality lead to a situation where resources are scarce. To deal with this resource limitation, platforms are commonly shared by multiple applications. For example, visual servoing [4] uses feedback information from visual sensors in motion control, where both image processing and control computation tasks are executed on the same processor. In such shared platforms, a scheduler statically/dynamically decides the availability of a resource to an application, and the order of execution of various tasks or applications. Realizing an equidistant sampling scheme in such shared embedded implementation imposes inflexibility and often leads to unnecessary expensive design solutions.

Recently, a potentially promising embedded platform candidate, Composable and Predictable System on Chip (CompSOC), was introduced [8]. Composability allows for independent development of multiple applications, while predictability provides precise temporal behavior of the platform. The CompSOC platform is suitable for independent development

Van Zundert, Oomen, and Heemels are with the Eindhoven University of Technology, Department of Mechanical Engineering, Control Systems Technology group, The Netherlands. Goswami is with the Eindhoven University of Technology, Department of Electrical Engineering, Electronic Systems group, The Netherlands. E-mail: j.c.d.v.zundert@tue.nl. and interference-free execution of (control) applications. In [14], it is shown that a resource efficient implementation of a control algorithm in such composable platform leads to a set of known, precise, and periodically varying sampling periods. Whereas the majority of control design techniques aims at a single sampling frequency, the aim of the present paper is to develop a control design framework that exploits the periodicity knowledge from the platform for analyzing and synthesizing motion controllers. In particular, the focus is on feedforward controllers, since they constitute the largest part of the motion system's control input [6].

The design of controllers for periodically time-varying systems has been investigated in [1], [18] and has been mainly applied to sampled-data designs with an equidistant sample frequency [2], [5]. These approaches have been further developed towards multi-rate sampling, where different actuator/sensor channels have different rates, see [10], [7], [13] for feedback designs, [11] for motion feedback control, and [17] for multi-rate feedforward design.

Although important developments for periodically timevarying systems have been developed, they are not directly applicable to feedforward design for a periodic sampling sequence. The main contribution of this paper is a framework for the design of feedforward controllers under periodic sampling. This combines the analysis of data-based feedforward design [15], [16], [3] with non-equidistant sampling, where the main technical step involves a specific lifting step.

The outline is as follows. First, the problem and control goal are formulated in section II. The model of the periodically time-varying sampled system is developed in section III. This model is used for feedforward controller design in section IV. In section $\mathrm{V}$, the advantages of describing and controlling the system as a time-varying sampled system instead of a conservative time-invariant sampled system are demonstrated through a simulation example. Finally, conclusions are given in section VI.

Notation Finite dimensional, linear, single-input, singleoutput, discrete-time systems are considered. Extension to multi-input, multi-output systems is straightforward, since the theory is based on state-space descriptions. Dotted lines indicate a high equidistant sampling rate, dashed lines a low equidistant sampling rate, and dash-dotted lines a timevarying sampling rate. Underlined variables indicate finitetime matrix descriptions. $I_{n}$ denotes the $n \times n$ identity matrix, $\otimes$ the Kronecker product, and $\circ$ the Hadamard product. The superscript 0 refers to the base period, subscript $i$ refers to subperiod $\Delta_{i}$. 


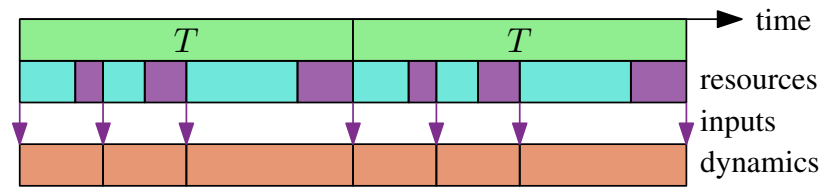

Fig. 1. Example timeline where the processor is allocated to either motion control tasks (cyan) or to non-motion control tasks (purple).

\begin{tabular}{|c|c|c|c|c|c|}
\hline \multicolumn{3}{|c|}{$T$} & \multicolumn{3}{c|}{$T$} \\
\hline$\Delta^{0}$ & $\Delta^{0}$ & $2 \Delta^{0}$ & $\Delta^{0}$ & $\Delta^{0}$ & $2 \Delta^{0}$ \\
\hline $2 \Delta^{0}$ & $2 \Delta^{0}$ & \multicolumn{2}{|c|}{$2 \Delta^{0}$} & $2 \Delta^{0}$ \\
\hline
\end{tabular}

Fig. 2. Example of periodically time-varying sampling where a period $T$ consists of three subperiods $\left(n_{\Delta}=3\right)$ with $K=[1,1,2]$.

\section{PROBlEM FORMULATION}

In this section, the objective is formulated by defining the periodic sampling sequence, the control configuration, and the control goal.

\section{A. Periodic sampling sequence}

In this section, the periodic timing behavior observed in platforms as [8], [14] is described. Such a platform runs under a time division multiplexing (TDM) policy where the TDM wheel of length $T$ is divided into a fixed number of time slots. Depending on the allocation of slots to the applications, the timing behavior of an application can be abstracted as shown in Fig. 1. The motion control task is only allocated and executed in the purple slots. Other applications run on the cyan slots. The composable nature of the platform allows for independent analysis of the control application.

Assumption 1 is imposed throughout.

Assumption 1. In a period $T$ there are $n_{\Delta}$ subperiods of length $\Delta_{i}, i=0,1, \ldots, n_{\Delta}-1$, which are an integer multiple of base period $\Delta^{0}$, i.e., $\Delta_{i}=k_{i} \Delta^{0}, k_{i} \in \mathbb{N}^{+}$.

An example of two periods $T$ is provided in Fig. 2, with the sampling sequence indicated by

$$
K:=\left[\begin{array}{llll}
k_{0} & k_{1} & \ldots & k_{n_{\Delta}-1}
\end{array}\right] .
$$

Note that there is more design freedom with the timevarying sequence (red) than with the conservative equidistant sampling sequence (yellow).

Remark 2. Assumption 1 can directly be relaxed at the expense of more involved derivations.

\section{B. Control configuration}

The motion system is controlled via the feedback/feedforward control architecture depicted in Fig. 3. The selected configuration is common in motion control, but the results can readily be extended to other configurations. Here, $P$ is the motion system, and $C_{F B}$ the feedback controller acting on the error error $\varepsilon$ between the output $\psi$ and the reference signal $\rho . C_{F F}$ is the feedforward controller to be designed, see section II-C.

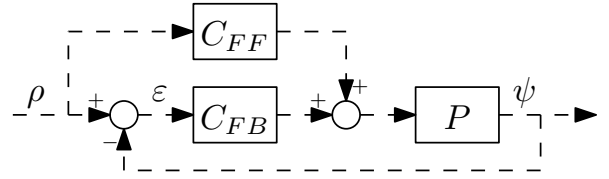

Fig. 3. Closed-loop control configuration.

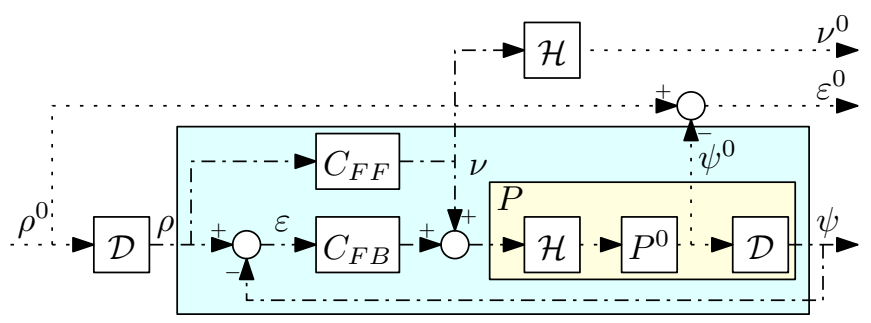

Fig. 4. Control diagram including the sample rate conversion between the equidistant rate $\Delta^{0}(-)$ and the time-varying rate $(-\cdot-\cdot)$. Only the part in blue is implemented on the actual system.

Including the periodically time-varying sampling into the control diagram of Fig. 3 yields Fig. 4. Here, $P=\mathcal{D} P^{0} \mathcal{H}$ is a sampled version of the (linear) plant $P^{0}$ at the base rate $\Delta^{0}$, with $\mathcal{D}$ and $\mathcal{H}$ the down- and upsampler, respectively, that are defined in section III-D.

\section{Control goal}

The control goal is to design the feedforward controller $C_{F F}$ such that error $\varepsilon$ is minimized according to a certain performance criterion. To provide a fair comparison, the feedback controller $C_{F B}$ is designed at a conservative, equidistant sampling rate, see also Fig 2. This is by no means restrictive and can directly be relaxed. The feedforward controller is explicitly designed and implemented at the time-varying rate. To enable a fair comparison, the tracking error $\varepsilon^{0}$ at equidistant rate $\Delta^{0}$ is used. This data is often available off-line and can be used in batch-to-batch feedforward control [9], [3]. The framework can easily be adapted for evaluation of the tracking error at other rates.

With the definition of the periodically time-varying sampling sequence and the control configuration, the main problem can be formulated, see Problem 3 .

Problem 3. Given the closed-loop configuration in Fig. 4, with stabilizing $C_{F B}$, and a periodically time-varying sampling sequence (see for example Fig. 2), determine the optimal feedforward controller

$$
C_{F F}^{o p t}:=\arg \min _{C_{F F} \in \mathcal{P}} V^{0}\left(C_{F F}\right),
$$

where

$$
V^{0}\left(C_{F F}\right)=\left\|\underline{\varepsilon}^{0}\right\|_{W_{\varepsilon}}^{2}+\left\|\underline{\nu}^{0}\right\|_{W_{\nu}}^{2},
$$

with $\|(\cdot)\|_{W}^{2}=(\cdot)^{\top} W(\cdot), W_{\varepsilon}^{\top}=W_{\varepsilon} \succ 0, W_{\nu}^{\top}=W_{\nu} \succeq$ 0 , and where $\varepsilon^{0}, \underline{\nu}^{0} \in \mathbb{R}^{N^{0}}, N^{0} \in \mathbb{N}^{+}$, are the lifted domain equivalents of $\varepsilon^{0}$ and $\nu^{0}$, respectively.

In section IV, the feedforward class $\mathcal{P}$ is defined and the optimal feedforward controller $C_{F F}^{o p t}$ is derived. The latter 


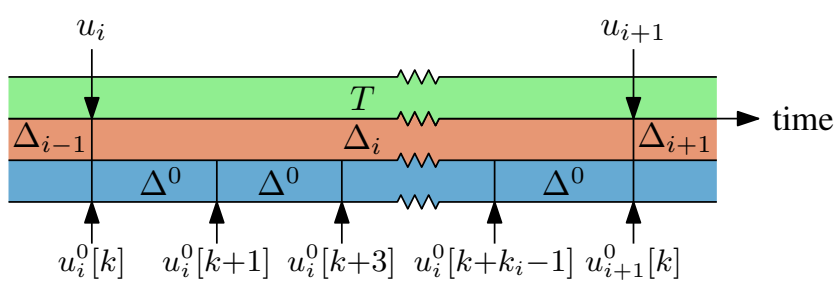

Fig. 5. A single period $\Delta_{i}$ consists of $k_{i}$ base periods $\Delta^{0}$ where the input $u_{i}^{0}$ remains constant.

requires the relation between $\underline{\nu}$ and $\underline{\varepsilon}^{0}$ which is derived next.

\section{SYSTEM DESCRIPTION}

In this section the time-varying system $P$ and feedback controller $C_{F B}$ are described in order to express $\underline{\varepsilon}^{0}$ in terms of $\underline{\nu}$. The design of $C_{F F}$ is presented separately in section IV. In the following sections, a systematic framework for describing these systems using finite-time descriptions is presented. In succession, the dynamics during a subperiod $\Delta_{i}$, during a period $T$, and during a finite length $N$ are described. Finally, finite-time descriptions of the down- and upsampler are derived, and the system interconnection is derived, providing the relation between $\underline{\varepsilon}^{0}$ and $\underline{\nu}$.

\section{A. Dynamics during a subperiod}

Due to the periodic nature, the system dynamics are identical for every period $T$. In order to describe the dynamics during a period $T$, a description of the dynamics during subperiods $\Delta_{i}$ is required. In Theorem 4 the dynamics over a subperiod are provided at rate $\Delta^{0}$. In Corollary 5 , the equivalent dynamics at rate $\Delta_{i}$ are presented.

Theorem 4. Let the dynamics of a discrete-time system with equidistant sampling time $\Delta^{0}$ have state-space representation $\left(A^{0}, B^{0}, C^{0}, D^{0}\right)$ and let the sampling periods $\Delta_{i}$ satisfy Assumption 1, see also Fig. 5. If a zero-orderhold of period $\Delta_{i}$ is applied to the input of this system, i.e., $u_{i}^{0}[k+n]=u_{i}[k], n=0,1, \ldots, k_{i}-1$, then the dynamics during the interval $\Delta_{i}$ are given by

$$
\begin{aligned}
x_{i}^{0}[k+n] & =\left(A^{0}\right)^{n} x_{i}^{0}[k]+\sum_{j=0}^{n-1}\left(A^{0}\right)^{j} B^{0} u_{i}^{0}[k], \quad n \leq k_{i}, \\
y_{i}^{0}[k] & =C^{0} x_{i}^{0}[k]+D^{0} u_{i}^{0}[k] .
\end{aligned}
$$

Proof. Follows from successive substitution.

Corollary 5. The equivalent dynamics of the system in Theorem 4 for sampling time $\Delta_{i}$ has state-space representation

$$
\left[\begin{array}{c|c}
A_{i} & B_{i} \\
\hline C_{i} & D_{i}
\end{array}\right]=\left[\begin{array}{c|c}
\left(A^{0}\right)^{k_{i}} & \sum_{j=0}^{k_{i}-1}\left(A^{0}\right)^{j} B^{0} \\
\hline C^{0} & D^{0}
\end{array}\right] .
$$

Corollary 5 shows that downsampling the system of Theorem 4 from sampling time $\Delta^{0}$ to $\Delta_{i}$ is equivalent to considering $n=k_{i}$ steps as a single step.

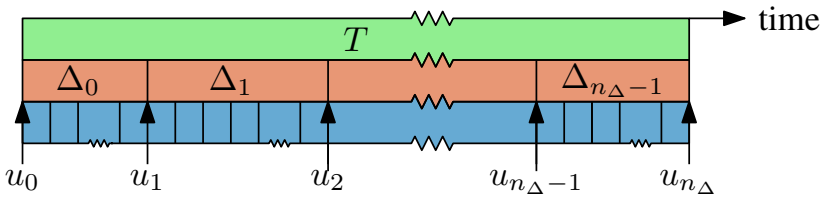

Fig. 6. The dynamics over a period $T$ is determined by the dynamics of the $n_{\Delta}$ subperiods $\Delta_{i}$.

\section{B. Dynamics during a period}

The dynamics during a subperiod at rate $\Delta_{i}$ are described by Corollary 5. By combining the dynamics of the $n_{\Delta}$ subperiods, see Fig. 6, the dynamics during a period $T$ are obtained as provided by Theorem 6 . Downsampling this system to rate $T$ yields a multi-input, multi-output system as shown by Theorem 7 .

Theorem 6. During period $T$, consisting of $n_{\Delta}$ periods $\Delta_{i}$, the dynamics at time-varying rate $\Delta_{i}$ evolve according to

$$
\begin{aligned}
& x_{n}=\prod_{j=1}^{n} A_{n-j} x_{0}+\sum_{i=0}^{n-1} \prod_{j=1}^{n-i-1} A_{n-j} B_{i} u_{i}, \\
& y_{n}=C_{n} x_{n}+D_{n} u_{n} .
\end{aligned}
$$

with $\prod_{j=1}^{n} A_{j}=I$ for $n<1$.

Proof. Follows from successive substitution of the dynamics in (2) according to Fig. 6.

Theorem 7. The dynamics of Theorem 6 at non-equidistant rate $\Delta_{i}$ have an $n_{\Delta}$-input, $n_{\Delta}$-output equivalent at equidistant rate $T$ with state-space realization

$$
\left[\begin{array}{c|cccc}
\sum_{j=1}^{n} A_{j} & \prod_{j=1}^{n} A_{j} B_{0} & \prod_{j=2}^{n} A_{j}^{-1} B_{1} & \cdots & B_{n^{-1}} \\
\hline C^{0} & D^{0} & 0 & \cdots & 0 \\
C^{0} A_{0} & C^{0} B_{0} & D^{0} & \ddots & \vdots \\
\vdots & \vdots & \ddots & \ddots & 0 \\
C^{0} \prod_{j=0}^{n} A_{j}^{-2} & C^{0} \sum_{j=1}^{n} A_{j} B_{0} & \cdots & B_{n} \Delta^{-2} & D^{0}
\end{array}\right],
$$

with state $x^{n_{\Delta}}=x_{k n_{\Delta}}(k \in \mathbb{N})$, and input $u^{n_{\Delta}}$ and output $y^{n_{\Delta}}$ given by

$$
u^{n_{\Delta}}=\left[\begin{array}{c}
u_{k n_{\Delta}} \\
u_{k n_{\Delta}+1} \\
\vdots \\
u_{(k+1) n_{\Delta}-1}
\end{array}\right], \quad y^{n_{\Delta}}=\left[\begin{array}{c}
y_{k n_{\Delta}} \\
y_{k n_{\Delta}+1} \\
\vdots \\
y_{(k+1) n_{\Delta}-1}
\end{array}\right] .
$$

Proof. Follows from successive substitution of the relations in Theorem 6.

Since the system is perceived at time-varying rate $\Delta_{i}$, Theorem 6 is used for deriving finite-time expressions in section III-C. Theorem 7 is used for feedforward controller design in section IV. 


\section{Finite-time description of the system}

The dynamics over the finite signal length are described using finite-time descriptions. First, finite-time descriptions for LTI systems are recapitulated. Second, finite-time expressions for the LPTV system are derived.

Let the single-rate discrete-time system $P \stackrel{s}{=}(A, B, C, D)$ be operating over a finite-time interval $[0, N-1]$. Then, the input-to-output behavior is given by

$$
\underline{\psi}=\underline{P} \underline{\nu}, \quad \underline{P}=\left[\begin{array}{ccccc}
p_{0} & 0 & 0 & \cdots & 0 \\
p_{1} & p_{0} & 0 & \cdots & 0 \\
p_{2} & p_{1} & p_{0} & \cdots & 0 \\
\vdots & \vdots & \vdots & \ddots & \vdots \\
p_{N-1} & p_{N-2} & p_{N-3} & \cdots & p_{0}
\end{array}\right],
$$

with Markov parameters $p_{0}=D$ and $p_{i}=C A^{i-1} B, i=$ $1,2, \ldots, N-1$. For causal SISO LTI systems, $\underline{P} \in \mathbb{R}^{N \times N}$ is a square lower triangular Toeplitz matrix that maps input vector $\underline{\nu}=\left[\begin{array}{lllll}\nu_{0} & \nu_{1} & \nu_{2} & \ldots & \nu_{N-1}\end{array}\right]^{\top} \in \mathbb{R}^{N}$ to output vector $\psi=\left[\begin{array}{lllll}\psi_{0} & \psi_{1} & \psi_{2} & \ldots & \psi_{N-1}\end{array}\right]^{\top} \in \mathbb{R}^{N}$.

Finite-time descriptions can also be used for LPTV systems. For time-invariant systems, entries in the finite-time description correspond to equidistant points in time. This property is lost for time-varying systems where the entries correspond to non-equidistant points in time determined by the sampling sequence $K$. The finite-time description for the LPTV system of Theorem 6 is provided by Theorem 8 .

Theorem 8. Given a state space realization $\left(A^{0}, B^{0}, C^{0}, D^{0}\right)$ of the system $P^{0}$ at equidistant rate $\Delta^{0}$, and a periodically time-varying sampling sequence of $n_{\Delta}$ subperiods per period $T$, the finite-time description of $P$, given the periodically time-varying sampling sequence $K$, is given by

$$
\underline{P}=\left[\begin{array}{ccccc:c}
D^{0} & 0 & 0 & \cdots & 0 & \cdots \\
C^{0} B_{0} & D^{0} & 0 & \cdots & 0 & \cdots \\
C^{0} A_{1} B_{0} & C^{0} B_{1} & D^{0} & \cdots & 0 & \cdots \\
\vdots & \ddots & \ddots & \ddots & \vdots & \ddots \\
C^{0} \prod_{j=2}^{n-1} A_{n_{\Delta}-j} B_{0} & \cdots & \cdots & C^{0} B_{n_{\Delta}-2} & D^{0} & \cdots \\
\hdashline \vdots & \ddots & \vdots & \vdots & \vdots & \ddots
\end{array}\right],
$$

where $A_{i}=\left(A^{0}\right)^{k_{i}}, B_{i}=\sum_{j=0}^{k_{i}-1}\left(A^{0}\right)^{j} B^{0}$, and $\underline{P} \in \mathbb{R}^{N \times N}$.

Proof. Due to space restrictions, the proof is omitted.

Note that $\underline{P}$ in Theorem 8 is block-Toeplitz with block size $n_{\Delta} \times n_{\Delta}$. The equidistant sampling case is a special case of Theorem 8, see Corollary 9.

Corollary 9. If $k_{i}=k$, $\forall i$, then $\left(A_{i}, B_{i}, C_{i}, D_{i}\right)=$ $(A, B, C, D), \forall i$, and the equidistant sampling case is recovered as a special case.

\section{Finite-time descriptions of rate conversions}

In Theorem 8, the finite-time description for the LPTV system is provided. To describe the full system of Fig. 4 in a finite-time framework, the finite-time descriptions of the downsampler $\mathcal{D}$ and zero-order-hold upsampler $\mathcal{H}$ are required. These are provided by Theorem 10 where it should be noted that these results can readily be extended to the situation when there is not an integer number of periods $T$ present in $N$.

Theorem 10. For the purpose of exposition, let the time span of $N$ samples consist of an integer number of periods $T$, and define the vectors

$$
\begin{aligned}
& \underline{\tau}_{T}^{0}[n]:=n-1, \quad n=1,2, \ldots, \frac{T}{\Delta^{0}}, \\
& \underline{\tau}_{T}[n]:= \begin{cases}0, & n=1, \\
\sum_{i=1}^{n-1} k_{i}, & n=2,3, \ldots, n_{\Delta}+1 .\end{cases}
\end{aligned}
$$

The finite-time expression of the downsampler $\mathcal{D}$ is

$$
\underline{\mathcal{D}}:=I_{\frac{N \Delta^{0}}{T}} \otimes \underline{\mathcal{D}}_{T},
$$

with $\underline{\mathcal{D}}_{T} \in \mathbb{R}^{n_{\Delta} \times \frac{T}{\Delta^{0}}}$ given by

$$
\underline{\mathcal{D}}_{T}(i, j):= \begin{cases}1, & \underline{\tau}_{T}[i]=\underline{\tau}_{T}^{0}[j] \\ 0, & \text { otherwise. }\end{cases}
$$

The finite-time expression of the zero-order-hold upsampler $\mathcal{H}$ is

$$
\underline{\mathcal{H}}:=I_{\frac{N^{0} \Delta^{0}}{T}} \otimes \underline{\mathcal{H}}_{T},
$$

with $\underline{\mathcal{H}}_{T} \in \mathbb{R}^{\frac{T}{\Delta^{0}} \times n_{\Delta}}$ given by

$$
\underline{\mathcal{H}}_{T}(i, j):= \begin{cases}1, & \underline{\tau}_{T}[j] \leq \underline{\tau}_{T}^{0}[i]<\underline{\tau}_{T}[j+1], \\ 0, & \text { otherwise. }\end{cases}
$$

Proof. See, for example, [12].

Note that both $\underline{\mathcal{H}}$ and $\underline{\mathcal{D}}$ are block-Toeplitz matrices. Furthermore, note that up-down conversion does not affect the signal $\left(\underline{\mathcal{D}} \underline{\mathcal{H}}=I_{N}\right)$, whereas down-up conversion does affect the signal $\left(\underline{\mathcal{H}} \underline{\mathcal{D}} \neq I_{N^{0}}\right)$.

\section{E. System interconnection}

By combining Theorem 8 and Theorem 10, the finite-time description of the system in Fig. 4 is complete and the system interconnection can be described. The error $\underline{\varepsilon}^{0}$ as function of the feedforward $\underline{\nu}$ is provided by Theorem 11 .

Theorem 11. The finite-time error $\underline{\varepsilon}^{0}$ in Fig. 4 for the equidistant rate $\Delta^{0}$ is given by

$$
\underline{\varepsilon}^{0}=\underline{S}^{0} \underline{\rho}^{0}-\underline{S}^{0} \underline{P}^{0} \underline{\mathcal{H}} \underline{\nu},
$$

with $\underline{S}^{0}=\left(I_{N^{0}}+\underline{P}^{0} \underline{\mathcal{H}} \underline{C}_{F B} \underline{\mathcal{D}}\right)^{-1}$.

Proof. The output at the base rate is given by

$$
\underline{\psi}^{0}=\underline{S}^{0} \underline{P}^{0} \underline{\mathcal{H}} \underline{\nu}+\underline{S}^{0} \underline{P}^{0} \underline{\mathcal{H}} \underline{C}_{F B} \underline{\mathcal{D}} \underline{\rho}^{0} .
$$

The result follows from substituting this expression in $\underline{\varepsilon}^{0}=$ $\underline{\rho}^{0}-\underline{\psi}^{0}$ and rearranging terms.

In this section, finite-time descriptions for the system interconnection of Fig. 4 are presented. Next, these expressions are used for designing the feedforward filter $C_{F F}$. 


\section{LIFTED DOMAIN FEEDFORWARD OPTIMIZATION}

In Problem 3, the feedforward controller $C_{F F}$ belongs to a class $\mathcal{P}$. This class is parameterized according to Definition 12. Parameter $\beta \in \mathbb{R}^{n_{\beta} n_{\Delta}^{2}}$ contains all parameters in $\underline{\beta}_{i}, i=0,1, \ldots, n_{\beta}-\overline{1}$.

Definition 12. The feedforward class $\mathcal{P}$ is given by

$$
\mathcal{P}=\left\{\sum_{i=0}^{n_{\beta}-1} \underline{\beta}_{i} \circ \vartheta_{i}(z) \mid \underline{\beta}_{i} \in \mathbb{R}^{n_{\Delta} \times n_{\Delta}}\right\},
$$

with $\vartheta_{i}(z)$ an $n_{\Delta}$-input, $n_{\Delta}$-output system of basis functions.

Note that the class $\mathcal{P}$ in Definition 12 consists of MIMO transfer functions in the so-called lifted domain [2]. In the physical time domain, after reversal of the lifting operator, it becomes a SISO yet LPTV operator, due to the periodic sampling sequence. Hence the name lifted feedforward controller. In future work, the choice of basis functions for such controllers is further explained.

The finite-time description of $C_{F F}$, denoted $\underline{C}_{F F}(\beta)$, depends on the particular choice of $K$. Since by Definition 12 it is linear in $\underline{\beta}$, there exists a matrix $\underline{T}_{\rho}^{\beta} \in \mathbb{R}^{N \times n_{\beta} n_{\Delta}^{2}}$ satisfying

$$
\underline{C}_{F F}(\underline{\beta}) \underline{\rho}=\underline{T}_{\rho}^{\beta} \underline{\beta} .
$$

With (3) and combining the results of the previous sections, the optimal feedforward filter can be computed, see Theorem 13.

Theorem 13. The optimal solution to Problem 3 with $\mathcal{P}$ according to Definition 12 is given by

$$
\begin{aligned}
\underline{\beta}^{\text {opt }} & =\left(\underline{\mathcal{M}}^{\top} W_{\varepsilon} \underline{\mathcal{M}}+\left(\underline{T}_{\rho}^{\beta}\right)^{\top} W_{\nu} \underline{T}_{\rho}^{\beta}\right)^{-1} \underline{\mathcal{M}^{\top}} W_{\varepsilon} \underline{b}, \\
\underline{b} & =\underline{S}^{0} \underline{\rho}^{0}, \\
\underline{\mathcal{M}} & =\underline{S}^{0} \underline{P}^{0} \underline{\mathcal{H}} \underline{T}_{\rho}^{\beta} .
\end{aligned}
$$

Proof. Substitution of $\underline{\nu}=\underline{C}_{F F}(\beta) \rho=\underline{T}_{\rho}^{\beta} \beta$ (see (3)) in Theorem 11 yields $\underline{\varepsilon}^{0}=\underline{b}-\underline{\mathcal{M}} \hat{\beta}$. Hence, $V^{\overline{0}}$ is quadratic in $\underline{\beta}$ and hence the minimum follows from $\nabla_{\underline{\beta}} V^{0}=0$.

Theorem 13 is used in the simulation case study of the next section.

\section{Simulation CASE Study}

Through use of a simulation case study, the advantage of the periodically time-varying sampling framework introduced in this paper over conservative equidistant sampling is shown.

\section{A. System definition}

The system is based on the rotational two-mass-springdamper system shown in Fig. a for which the Bode magnitude plot is shown in Fig. b. The feedback controller $C_{F B}$ is a lead filter yielding a closed-loop bandwidth of $10 \mathrm{~Hz}$. In order to have the same feedback controller for each sampling sequence, the feedback controller is designed at the lowest rate. The reference signal $\rho^{0}$ is selected as the fourth order point-to-point trajectory depicted in Fig. 8.

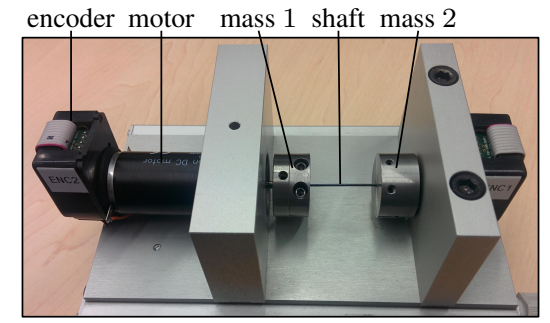

(a) Photograph of the mechanical setup consisting of two masses interconnected by a flexible shaft.

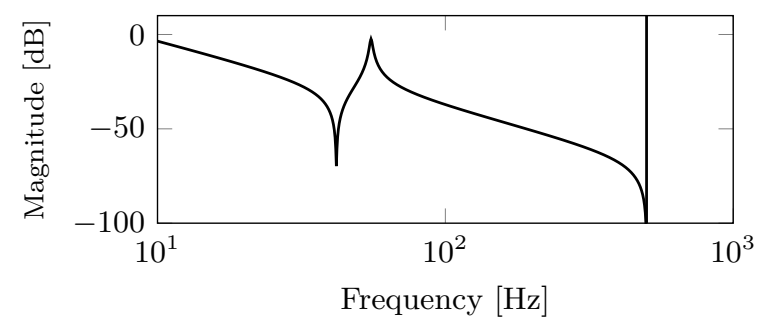

(b) Bode magnitude plot of the model for sampling time $\Delta^{0}=$ $1 \mathrm{~ms}$.

Fig. 7. The system $P$ is the model of the collocated control loop from the motor to the encoder.

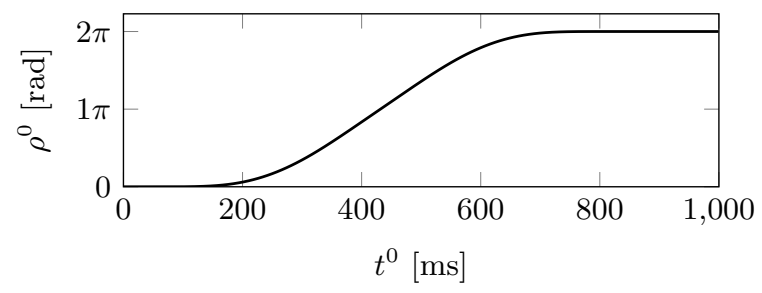

Fig. 8. The reference trajectory is a fourth order point-to-point profile.

\section{B. Sampling sequences}

In this case study, the sampling sequence of Fig. 2 is used, i.e. $K=[1,1,2]$, see also Fig. 9. The highest possible equidistant sampling rate is $2 \Delta^{0}$, which is conservative since in each period $T$ a control point is neglected. The proposed framework allows to exploit all possible control points. Since this increases the freedom of the feedforward signal, an increase in performance can be expected.

The basis functions $\vartheta_{i}(z)$ in Definition 12 are selected as

$$
\vartheta_{i}(z)=z^{-i}\left[\begin{array}{cccc}
1 & 1 & \cdots & 1 \\
1 & 1 & \cdots & 1 \\
\vdots & \vdots & \ddots & \vdots \\
1 & 1 & \cdots & 1
\end{array}\right]
$$

In the simulation $\Delta^{0}=0.001 \mathrm{~s}, N^{0}=1000$, and the weights in (1) are selected as $W_{\varepsilon}=10^{12} I_{N^{0}}$ and $W_{\nu}=0_{N^{0}}$ in order to minimize $\underline{\varepsilon}^{0}$.

\section{Results}

For comparison, the results for sampling at the base rate $\Delta^{0}$ are also presented. Note that this is typically not possible in practice, but included here as benchmark. The results are shown in Fig. 10.

The performance metric $V^{0}$ as function of $n_{\beta}$ for the three sampling sequences is shown in Fig. a. A higher $n_{\beta}$ means a 


\begin{tabular}{|c|c|c|c|c|c|c|c|}
\hline \multicolumn{4}{|c|}{$T$} & \multicolumn{4}{c|}{$T$} \\
\hline$\Delta^{0}$ & $\Delta^{0}$ & $\Delta^{0}$ & $\Delta^{0}$ & $\Delta^{0}$ & $\Delta^{0}$ & $\Delta^{0}$ & $\Delta^{0}$ \\
\hline$\Delta^{0}$ & $\Delta^{0}$ & $2 \Delta^{0}$ & $\Delta^{0}$ & $\Delta^{0}$ & $2 \Delta^{0}$ \\
\hline \multicolumn{2}{|c|}{$2 \Delta^{0}$} & \multicolumn{2}{|c|}{$2 \Delta^{0}$} & \multicolumn{2}{|c|}{$2 \Delta^{0}$} & $2 \Delta^{0}$ \\
\hline
\end{tabular}

Fig. 9. Two periods $T$ of the case study's timeline with the base rate $\Delta^{0}$ (blue), time-varying sampling (red), and equidistant sampling (yellow).

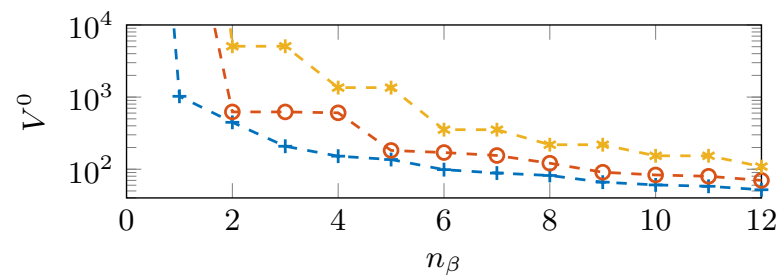

(a) Performance metric $V^{0}$ vs. number of parameterized periods $n_{\beta}$.

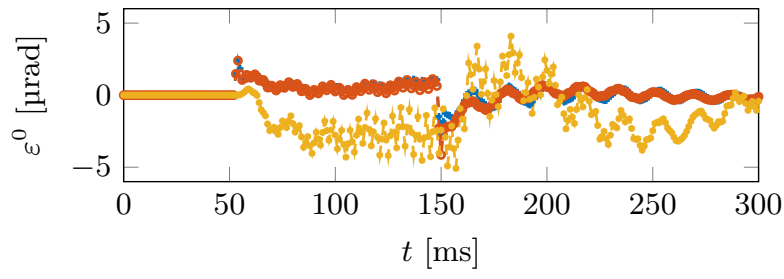

(b) Error signal $\varepsilon^{0}$ at the start of the motion for $n_{\beta}=3$.

Fig. 10. Results of the case study for $K=[1,1,1,1](\cdot+\cdot), K=[1,1,2]$ $(\cdot \bullet \cdot)$, and $K=[2,2](\cdot \bullet)$.

larger operating timespan of the feedforward controller and therefore an improved performance. This is indeed observed for all three cases: $V^{0}$ decreases for increasing $n_{\beta}$. As was expected, the performance of the sampling sequence $K=$ $[1,1,2]$ is worse than for $K=[1,1,1,1]$ (less control points per period) and higher than for $K=[2,2]$ (more control points per period). The time domain error signal $\underline{\varepsilon}^{0}$ near the start of the motion is provided in Fig. $\mathrm{b}$ for $n_{\beta}=3$.

\section{CONCLUSIONS}

A resource-efficient implementation on a class of predictable platforms leads to a periodically switched system due to periodic non-uniform sampling periods. The analysis and controller design of such systems can be done by I) settling with slower equidistant sampling of the system and using standard LTI techniques, but this is often conservative in terms of performance since not all measurement and actuation points are exploited; or II) controlling the system as a periodically time-varying system and exploiting all possible control points. In this paper a framework is introduced that allows to describe the periodically time-varying systems of option II). Moreover, the framework allows for optimal feedforward design incorporating the time-varying sampling of the system. As a case study, a motion control application is considered and through simulation it is shown that timevarying sampling control of solution II) is indeed superior to conservative equidistant sampling of solution I).

Ongoing work focuses on experimental validation of the presented work, optimal selection of the sampling sequence, and design of feedback control, rational feedforward, and ILC for periodically time-varying systems.

\section{ACKNOWLEDGMENTS}

The authors would like to acknowledge the contributions of Jan Verhaegh, Wouter Aangenent, and Duarte Antunes to initial research, and the fruitful discussions with Zhang Ji, Juan Valencia Payan, and Martijn Koedam that have contributed to the results presented in this paper.

This research is supported by the Dutch Technology Foundation STW, carried out as part of the Robust Cyber-Physical Systems (RCPS) project (no. 12694); and Innovational Research Incentives Scheme under the VENI grant "Precision Motion: Beyond the Nanometer" (no. 13073) awarded by NWO (The Netherlands Organization for Scientific Research).

\section{REFERENCES}

[1] Bassam Bamieh, J. Boyd Pearson, Bruce A. Francis, and Allen Tannenbaum. A lifting technique for linear periodic systems with applications to sampled-data control. Systems \& Control Letters, 17(2):79-88, 1991.

[2] Bassam A. Bamieh and J. Boyd Pearson Jr. A General Framework for Linear Periodic Systems with Applications to $H_{\infty}$ Sampled-Data Control. Transactions on Automatic Control, 37(4):418-435, 1992.

[3] Frank Boeren, Tom Oomen, and Maarten Steinbuch. Iterative motion feedforward tuning: A data-driven approach based on instrumental variable identification. Control Engineering Practice, 37:11-19, 2015.

[4] François Chaumette and Seth Hutchinson. Visual Servo Control Part I: Basic Approaches. Robotics and Automation Magazine, 13(4):82-90, 2006.

[5] Tongwen Chen and Bruce A. Francis. Optimal Sampled-Data Control Systems. Springer, London, Great Britain, 1995.

[6] Edgar de Gelder, Marc van de Wal, Carsten Scherer, Camile Hol, and Okko Bosgra. Nominal and Robust Feedforward Design With Time Domain Constraints Applied to a Wafer Stage. Journal of Dynamic Systems, Measurement, and Control, 128(2):204-215, 2006.

[7] Hiroshi Fujimoto and Yoichi Hori. High-performance servo systems based on multirate sampling control. Control Engineering Practice, 10(7):773-781, 2002.

[8] Kees Goossens, Arnaldo Azevedo, Karthik Chandrasekar, Manil Dev Gomony, Sven Goossens, Martijn Koedam, Yonghui Li, Davit Mirzoyan, Anca Molnos, Ashkan Beyranvand Nejad, Andrew Nelson, and Shubhendu Sinha. Virtual Execution Platforms for Mixed-TimeCriticality Systems: The CompSOC Architecture and Design Flow. ACM SIGBED Review, 10(3):23-34, 2013

[9] Dimitry Gorinevsky. Loop Shaping for Iterative Control of Batch Processes. Control Systems Magazine, 22(6):55-65, 2002.

[10] Sanjay Lall and Geir Dullerud. An LMI solution to the robust synthesis problem for multi-rate sampled-data systems. Automatica, 37(12):1909-1922, 2001.

[11] T. Oomen, M. van de Wal, and O. Bosgra. Design framework for high-performance optimal sampled-data control with application to a wafer stage. International Journal of Control, 80(6):919-934, 2007.

[12] Tom Oomen, Jeroen van de Wijdeven, and Okko Bosgra. Suppressing intersample behavior in iterative learning control. Automatica, 45(4):981-988, 2009.

[13] Julián Salt and Pedro Albertos. Model-Based Multirate Controllers Design. Transactions on Control Systems Technology, 13(6):988-997, 2005.

[14] Juan Valencia, Dip Goswami, and Kees Goossens. Composable Platform-Aware Embedded Control Systems on a Multi-Core Architecture. In Proceedings of Euromicro Symposium on Digital System Design (DSD), 2015.

[15] J. van de Wijdeven and O.H. Bosgra. Using basis functions in iterative learning control: analysis and design theory. International Journal of Control, 83(4):661-675, 2010.

[16] Stan H. van der Meulen, Rob L. Tousain, and Okko H. Bosgra. Fixed Structure Feedforward Controller Design Exploiting Iterative Trials: Application to a Wafer Stage and a Desktop Printer. Journal of Dynamic Systems, Measurement, and Control, 130(5):051006:1-16, 2008. 
[17] J.C.D. Van Zundert, J.L.C. Verhaegh, W.H.T.M. Aangenent, T. Oomen, D. Antunes, and W.P.M.H. Heemels. Feedforward for Multi-Rate Motion Control: Enhanced Performance and Cost-Effectiveness. In Proceedings of the 2015 American Control Conference, volume 2015July, pages 2831-2836, Chicago, Illinois, 2015.

[18] Yutaka Yamamoto and Pramod P. Khargonekar. Frequency Response of Sampled-Data Systems. Transactions on Automatic Control, 41(2):166-176, 1996. 